\title{
Selective Thoracic Fusion for King-Moe Type II/Lenke 1C Curve in Adolescent Idiopathic Scoliosis: A Comprehensive Review of Major Concerns
}

\author{
Masayuki Ishikawa1), Makoto Nishiyama ${ }^{2)}$ and Michihiro Kamata ${ }^{1)}$ \\ 1) Department of Orthopaedic Surgery, Keiyu Hospital, Kanagawa, Japan \\ 2) Spine and Spinal Cord Center, Mita Hospital, International University of Health and Welfare, Tokyo, Japan
}

\begin{abstract}
:
Introduction: Controversies still exist in the surgical indications and outcomes of selective thoracic fusion (STF) for a primary thoracic curve with a compensatory large lumbar curve (King-Moe type II/Lenke 1C curve) in adolescent idiopathic scoliosis (AIS). Issues of the greatest concern regarding this curve type include curve criteria that indicate STF to prevent postoperative coronal decompensation and postoperative radiographic outcomes, including curve correction, coronal balance, and thoracolumbar kyphosis, after STF.
\end{abstract}

Methods: This review comprehensively documents the issues raised in the literature regarding surgical indications and radiographic outcomes of STF for King-Moe type II/Lenke 1C curve in AIS.

Results: Studies suggest that radiographic curve criteria indicating STF for this curve type include the preoperative dominance of the thoracic curve to the lumbar curve in the Cobb angle and the characteristics of the lumbar curve in magnitude and flexibility. Studies warn the need for a careful clinical evaluation of the thoracic and lumbar rotational prominences. Documented radiographic outcomes of importance include the postoperative behavior of the unfused lumbar curve, coronal or sagittal decompensation after STF, and factors associated with these issues.

A comprehensive review of the literature suggests that the use of a segmental pedicle screw construct and better instrumented thoracic curve correction achieve better spontaneous lumbar curve correction. Although the causes of postoperative coronal decompensation remain multifactorial, preoperative coronal decompensation to the left and an inappropriate selection of the lowest instrumented vertebra are consistently reported to be the major causative factors.

Conclusions: STF has been validated in general for the treatment of King-Moe type II or Lenke 1C curve in AIS; however, controversies remain regarding the surgical indications and outcomes.

Long-term impacts of residual lumbar curve, coronal decompensation, and mild thoracolumbar kyphosis on clinical outcomes after STF, along with optimal indications and strategy for STF, should further be assessed.

Keywords:

Adolescent idiopathic scoliosis, King type II curve, Lenke 1C curve, Selective thoracic fusion, Surgical indication, Spontaneous correction, Coronal decompensation, Thoracolumbar kyphosis

Spine Surg Relat Res 2019; 3(2): 113-125 dx.doi.org/10.22603/ssrr.2018-0047

\section{Introduction}

Adolescent idiopathic scoliosis (AIS) is a threedimensional spinal deformity. Surgical intervention is usually indicated if the primary curve exceeds $45^{\circ}-50^{\circ}$ because the long-term natural history of untreated idiopathic scoliosis dictates that such curves progress even after reaching skeletal maturity ${ }^{1-4)}$. The untreated, progressed spinal deform- ity can cause severe trunk deformity, decreased pulmonary function, and disabling low back pain. Surgical intervention with spinal instrumentation and fusion can correct the spinal deformity and achieve the cessation of curve progression, and most studies have shown satisfactory long-term radiographic and clinical outcomes ${ }^{5-8)}$. However, several long-term follow-up studies on postoperative AIS patients have demonstrated that spinal fusion to the middle or lower lumbar

Corresponding author: Masayuki Ishikawa, masa2001@ rb4.so-net.ne.jp

Received: July 8, 2018, Accepted: July 25, 2018, Advance Publication: October 10, 2018

Copyright (C) 2019 The Japanese Society for Spine Surgery and Related Research 
Table 1. Reported Radiographic Criteria of STF for King-Moe II/Lenke 1C Curve.

\begin{tabular}{|c|c|c|}
\hline Author (Year) & Constructs & Criteria \\
\hline King (1983) & HRI & $\mathrm{T}$ Cobb $\geq$ L Cobb, F.I. $\geq 0$ \\
\hline Lenke (1992) & CDI & $\begin{array}{c}\text { T:L Cobb ratio }>1.2, \text { T:L AVT ratio }>1.2, \text { T:L AVR ratio }>1.0, \\
\text { L Cobb }<60^{\circ}, \mathrm{L} \text { AVR }<\text { N-M } 2.5, \text { L AVT }<4.0 \mathrm{~cm}\end{array}$ \\
\hline Richards (1992) & CDI, TSRH & $\mathrm{L} \mathrm{Cobb}<40^{\circ}$ \\
\hline McCall (1992) & CDI & L Cobb $<45^{\circ}$, F.I. $>25$ \\
\hline Lenke (2003) & Modern segmental system & $\begin{array}{c}\text { T: } \mathrm{L} \text { Cobb ratio }>1.2, \mathrm{~T}: \mathrm{L} \text { AVT ratio }>1.2, \mathrm{~T}: \mathrm{L} \text { AVR ratio }>1.2, \\
\text { Bending L Cobb }<25^{\circ}, \mathrm{T} 10-\mathrm{L} 2 \text { kyphosis }<10^{\circ}\end{array}$ \\
\hline Majd (2003) & Modern segmental system & L Flexibility $>50 \%$, Bending L Cobb $<30^{\circ}$ \\
\hline Qiu (2005) & Modern segmental system & $\begin{array}{c}\text { T Cobb }>\text { L Cobb }-10^{\circ}, \text { Flexibility } \mathrm{L}>\mathrm{T}, \mathrm{L} \text { Cobb } \leq 45^{\circ}, \\
\text { L Flexibility } \geq 70 \%, \text { L AVR }<\text { N-M } 2, \text { T12-L1 kyphosis } \leq 0^{\circ}\end{array}$ \\
\hline Chang (2014) & PS & Lenke $1234 \mathrm{C}$, Bending L Cobb $<45^{\circ}, \mathrm{T} 10-\mathrm{L} 2$ kyphosis $<20^{\circ}$ \\
\hline
\end{tabular}

STF, selective thoracic fusion; HRI, Harrington rod instrumentation; T, thoracic; L, lumbar; F.I., flexibility index; CDI, CotrelDubousset instrumentation; AVT, apical vertebral translation; AVR, apical vertebral rotation; N-M, Nash-Moe; TSRH, Texas Scottish Rite Hospital; PS, pedicle screw

spine may have adverse effects, including an early degeneration of the unfused lumbar spine below the fusion mass with or without low back pain ${ }^{4,6,7,-15)}$. Therefore, the surgical strategy should aim for maximal three-dimensional spinal deformity correction with a solid arthrodesis while maintaining coronal and sagittal balance, sparing more mobile segments, and avoiding complications. In cases involving significant thoracic and lumbar curves that completely deviate from the midline, whether a selective fusion of the major curve or a fusion of both the thoracic and lumbar curves should be performed remains controversial.

After selective thoracic fusion (STF) for a primary thoracic curve with a compensatory large lumbar curve (now known as a King-Moe type II curve or Lenke 1C curve) was advocated by Moe in $1958^{16}$, numerous reports regarding surgical indications and outcomes of STF for this curve type have been published ${ }^{17-29)}$. While STF is still considered the gold standard for treating this curve type, non-STF, which corrects and fuses both the thoracic and lumbar curves, often necessitating a long spinal fusion to L3 or L4, has also been performed by numerous surgeons ${ }^{30-32}$. Such surgery is undertaken because of the shortcomings of STF, including residual or progressive lumbar curve and coronal or sagittal decompensation after surgery. Thus, several studies on STF for this curve type have focused on radiographic and clinical criteria that make a curve amenable to STF to yield optimal outcomes ${ }^{17-29,33,34)}$.

Radiographic outcomes after STF have been primarily based on the postoperative behavior of the unfused lumbar curve and coronal balance for various surgical approaches or constructs. Spontaneous lumbar curve correction (SLCC), coronal or sagittal decompensation after STF, and their causative factors are major concerns in treating this type of a curve (8,25-28,35-48) $^{\text {. }}$

With the evolution of spinal implants, devices, and corrective maneuvers and the accumulation of data on the nature of spinal deformity, surgical indications and outcomes of STF have changed. However, the ultimate goals of surgical treatment remain the same, and the efficacy of STF should be determined by long-term radiographic, cosmetic, and patient-reported outcomes.

This review of the literature aims to increase the surgeons' understanding of our current knowledge of STF with the goal of improving outcomes. Here we comprehensively document the major concerns regarding the use of STF for treating the King-Moe type II/Lenke 1C curve in AIS, including surgical indications and postoperative radiographic outcomes of curve correction, coronal balance, and thoracolumbar kyphosis.

\section{Radiographic Criteria for STF}

In 1958, Moe first introduced the concept of STF for a primary thoracic curve with a compensatory lumbar curve and stated that the characteristic of the curve pattern amenable to STF was the primary right thoracic curve with a left lumbar curve, being somewhat structural but not as inflexible as a thoracic curve with bending to the side ${ }^{16}$. This concept was a milestone in treating the King-Moe type II/Lenke $1 \mathrm{C}$ curve and was subsequently followed by several studies on surgical indications (Table 1). However, clear thresholds on the magnitude or flexibility of curves suitable for STF were not stated in his article.

In 1983, King and Moe advanced Moe's original idea of STF, stating that a King-Moe type II curve, in which both the thoracic and lumbar curves cross the midline and thoracic curve is equal to or larger than a lumbar curve with the flexibility of a lumbar curve being larger than that of a thoracic curve (positive flexibility index), can be successfully treated by STF with the lowest instrumented vertebra (LIV) at the neutral (NV) and stable (SV) vertebra using the Harrington rod instrumentation (HRI) ${ }^{17,49,50)}$. The King-Moe classification of thoracic idiopathic scoliosis was a longstanding, widely accepted system used to classify curve patterns and to recommend fusion levels. However, this system is limited in that the classification is based on the coronal plane only and has relatively poor to fair intra- and interobserver reliabilities ${ }^{21}$. 
In the early 1980's, Cotrel-Dubousset instrumentation (CDI) was introduced in AIS treatment ${ }^{51)}$. Thereafter, several reports on surgical outcomes of STF using this system for King-Moe type II curve have indicated the significant problem of postoperative coronal decompensation despite the implementation of the King-Moe's rule on LIV selection, leading to the development of several criteria for STF to prevent postoperative coronal decompensation ${ }^{18-20,35,37)}$.

In 1992, Lenke et al. stated that the King-Moe criteria for STF (thoracic curve $\geq$ lumbar curve and positive flexibility index) seemed to work quite well for HRI but were not sufficient when using CDI. This conclusion was drawn on the basis of a retrospective radiographic review of STF for the King-Moe type II curves, including revision cases for postoperative coronal decompensation. More strict criteria for STF with CDI were proposed, including the ratios of the thoracic curve to the lumbar curve in the Cobb angle, apical vertebral translation (AVT), and apical vertebral rotation (AVR). They suggested that 2 or 3 ratios of thoracic to lumbar curves (Cobb angle ratio $>1.2$, AVT ratio $>1.2$, AVR ratio $>1.0$ ) should be fulfilled to obtain successful outcomes after STF; otherwise, postoperative coronal decompensation would be more likely to occur. In addition, curves not meeting the ratio criteria or those with lumbar curves $>60^{\circ}$, Nash-Moe's rotation grade $>2.5$, or AVT $>4.0 \mathrm{~cm}$ should be treated as a double major curve (non-STF) ${ }^{18)}$.

In 1992, McCall and Bronson reported the surgical outcomes of STF using CDI for the King-Moe type II curve and determined the causative factors for postoperative coronal decompensation. Based on their evaluation, they provided criteria for STF using CDI, including a lumbar curve of $<45^{\circ}$ and a flexibility index of $>25$ to prevent postoperative coronal decompensation ${ }^{20)}$.

In 2001, Lenke et al. published the AIS classification system, which combines six coronal curve patterns (1-6) with three lumbar modifiers (A, B, or C) and three sagittal thoracic modifiers $(-, \mathrm{N}$, or +$)$. They stated that STF could be used to treat Lenke $1 \mathrm{C}$ curve that meets the following criteria: 1) the main thoracic curve is the major curve (the greatest Cobb magnitude); 2) the thoracolumbar/lumbar curve is a compensatory curve, completely crossing the center sacral vertical line and decreasing to $<25^{\circ}$ on side-bending; and 3) thoracolumbar kyphosis $\left(\right.$ T10-L2) $<20^{\circ 21,52)}$. Subsequently, they further speculated that patients with a Lenke $1 \mathrm{C}$ curve that meets the thoracic to lumbar curve ratio criteria (Cobb angle ratio $>1.2$, AVT ratio $>1.2$, and AVR ratio $>1.2$ ) with thoracolumbar kyphosis of $<10^{\circ}$ are more successfully treated by anterior or posterior STF with a modern segmental system ${ }^{22)}$

In 2007, Chang et al. showed surgical results of STF using a cantilever bending technique and direct vertebral rotation with pedicle screw (PS) construct for Lenke 1C and 2C curves meeting the Lenke's ratio criteria for STF, in which SLCC exceeds the flexibility of the original preoperative lumbar curve. With the success of this technique for enhancing the capacity of the lumbar curve for spontaneous correc- tion, they subsequently broadened the indication for STF to all Lenke $1 \mathrm{C}$ and $2 \mathrm{C}$ curves, regardless of whether they met the Lenke's ratio criteria, and some Lenke 3C and $4 \mathrm{C}$ curves in which the preoperative lumbar curves were $<45^{\circ}$ on side-bending without thoracolumbar kyphosis of $\geq 20^{\circ 25-28)}$.

In 2014, Schulz et al. published optimal postoperative radiographic coronal parameters after STF for Lenke 1, 2, 3, and $4 \mathrm{C}$ curves with queried data, surgeons' opinions, and patients-reported clinical outcomes, including the residual lumbar Cobb angle $<26^{\circ}$, lumbar curve correction rate $>$ $37 \%$, coronal balance $\leq 2 \mathrm{~cm}$, trunk shift $<1.5 \mathrm{~cm}$, and a deformity-flexibility quotient $(\mathrm{DFQ})<4^{53}$. They found that patients with a preoperative lumbar curve $<45^{\circ}$ or preoperative bending lumbar Cobb angle $<25^{\circ}$ achieve optimal postoperative coronal parameters for the lumbar Cobb angle and DFQ after STF, whereas those with thoracic to lumbar curve ratios $>1.2$ in the Cobb angle and AVT do not achieve optimal postoperative coronal parameters ${ }^{29)}$.

Thus, the reported radiographic criteria of STF for this curve type have attempted to optimize the residual unfused lumbar curve size and prevent postoperative coronal decompensation. However, no definite criteria still exist. Further studies to determine more predictable and reproducible criteria for STF using recent three-dimensional imaging techniques are warranted to optimize SLCC and to avoid postoperative coronal decompensation in treating King-Moe type II or Lenke $1 \mathrm{C}$ curves.

Table 2 demonstrates the reported incidence of STF and preoperative radiographic measurements of STF vs. non-STF for treating this curve type. A considerable number of patients are still treated with non-STF, despite the original authors' recommendation to use STF for King-Moe type II or Lenke $1 \mathrm{C}$ curves. A larger Cobb angle or AVT of a lumbar curve and smaller ratios of the thoracic curve to the lumbar curve in the Cobb angle, AVT and AVR drive a surgeon toward choosing non-STF ${ }^{10,31,32,37)}$.

The original term "STF" describes the fusion of only the thoracic curve in patients with a primary thoracic curve with a compensatory lumbar ' $C$ ' modifier curve ${ }^{17,22,54)}$. The majority of reports prefer to define an STF as LIV selected at L1 or above, whereas non-STF is considered to be a long spinal fusion beyond the lumbar apex, with the most common LIV being L3 (Table 2, 3).

\section{Clinical Criteria for STF}

King cautioned the importance of the careful clinical evaluation of appearance before surgical intervention, and stated that to be classified as a King-Moe type II curve, the thoracic rotational prominence should be characteristically larger than the lumbar rotational prominence during the forward-bending test ${ }^{31,33,34)}$. Lenke et al. proposed clinical criteria for STF, including 1) right shoulder elevation or leveled shoulders, 2) thoracic trunk shift > lumbar waistline asymmetry, and 3) thoracic to lumbar prominence ratio in scoliometer $\geq 1.2^{222}$. 
Table 2. Reported Incidence of STF and Preoperative Radiographic Measurements of STF vs. Non-STF for King-Moe II/ Lenke $1 \mathrm{C}$ Curve.

\begin{tabular}{|c|c|c|c|c|c|c|c|}
\hline Author (Year) & Curve type & Institute & $\begin{array}{c}\mathrm{N} \\
(\mathrm{STF}+\text { non-STF) }\end{array}$ & Parameters & STF & Non-STF & $\mathrm{P}$ value \\
\hline \multirow[t]{2}{*}{ King (1983) } & King-Moe II & Multi-center & 132 & Incidence (\%) & 84 & 16 & - \\
\hline & & & & LIV & NA & NA & - \\
\hline \multirow[t]{5}{*}{ Richards (1994) } & King-Moe II & Single-center & 35 & Incidence (\%) & 46 & 54 & - \\
\hline & & & & LIV & NA & NA & - \\
\hline & & & & L Cobb $\left(^{\circ}\right)$ & 46 & 54 & NA \\
\hline & & & & Bend L Cobb $\left(^{\circ}\right)$ & 13 & 18 & NA \\
\hline & & & & L Flexibility (\%) & 73 & 67 & NA \\
\hline \multirow[t]{2}{*}{ Lenke (2002) } & Lenke 1C & Multi-center & 65 & Incidence (\%) & 62 & 38 & - \\
\hline & & & & LIV & NA & NA & - \\
\hline \multirow[t]{6}{*}{ Newton (2003) } & Lenke 1B, C & Multi-center & 203 & Incidence for Lenke $1 \mathrm{C}(\%)$ & 68 & 32 & - \\
\hline & & & & LIV & $\geq \mathrm{L} 1$ & $\leq \mathrm{L} 2$ & - \\
\hline & & & & L Cobb $\left(^{\circ}\right)$ & 37 & 42 & 0.003 \\
\hline & & & & Bend L Cobb $\left(^{\circ}\right)$ & 10 & 13 & 0.02 \\
\hline & & & & L AVT (mm) & 22 & 31 & $<0.001$ \\
\hline & & & & $\mathrm{T} / \mathrm{L}$ Cobb ratio & 1.44 & 1.31 & 0.01 \\
\hline \multirow[t]{8}{*}{ Crawford (2013) } & Lenke 1C & Multi-center & 264 & Incidence (\%) & 49 & 51 & - \\
\hline & & & & LIV & $\geq \mathrm{L} 1$ & $\mathrm{~L} 3, \mathrm{~L} 4$ & - \\
\hline & & & & L Cobb $\left(^{\circ}\right)$ & 42.1 & 47 & $<0.01$ \\
\hline & & & & T AVT (mm) & 45.7 & 39.9 & $<0.01$ \\
\hline & & & & L AVT (mm) & 26.1 & 32.3 & $<0.01$ \\
\hline & & & & $\mathrm{T} / \mathrm{L}$ Cobb ratio & 1.35 & 1.18 & $<0.01$ \\
\hline & & & & $\mathrm{T} / \mathrm{L}$ AVT ratio & 1.82 & 1.31 & $<0.01$ \\
\hline & & & & $\mathrm{T} / \mathrm{L}$ AVR ratio & 1.15 & 0.98 & $<0.01$ \\
\hline \multirow[t]{2}{*}{ Demura (2013) } & Lenke 1C & Multi-center & 71 & Incidence (\%) & 75 & 25 & - \\
\hline & & & & LIV & $>\mathrm{L} 1$ & NA & - \\
\hline \multirow[t]{2}{*}{ Chang (2014) } & Lenke 1C & Single-center & 84 & Incidence (\%) & 98 & 2 & - \\
\hline & & & & LIV & NA & NA & - \\
\hline
\end{tabular}

STF, selective thoracic fusion; LIV, lowest instrumented vertebra; NA, not available; L, lumbar; AVT, apical vertebral translation; T, thoracic; AVR, apical vertebral rotation

The careful clinical evaluation of the thoracic and lumbar rotational prominences is greater emphasized in thoracic and lumbar curves of comparable Cobb magnitude and AVT on a preoperative postero-anterior radiograph, indicating similar structural characteristics in the thoracic and lumbar curves ${ }^{55)}$. One possible reason for the increased rate of non-STF for Lenke $1 \mathrm{C}$ curves may be the significant lumbar prominence, implicating a potential limitation of the two-dimensional Lenke's classification $^{32)}$.

\section{Curve Correction}

Curve correction rates for instrumented thoracic and unfused lumbar curves after STF for this curve type are reported as $24 \%-83 \%$ and $21 \%-81 \%$, respectively (Table 3 ).

In the HRI era, the instrumented thoracic curve correction was approximately equal to or slightly less than the preoperative thoracic curve flexibility on side-bending ${ }^{17,34,56-59)}$. In contrast, thoracic curve correction exceeds preoperative thoracic curve flexibility in modern segmental systems; however, SLCC does not usually exceed the preoperative lumbar curve flexibility ${ }^{7,20,25-28,35,47,48,60-66)}$. In the CDI era, an overcorrection of the thoracic curve in excess of the preoperative thoracic curve flexibility using a derotation maneuver was considered to be a causative factor of postoperative coronal decompensation. Thus, some reported curve correction rates using this system are somewhat low because of the surgeons' intentional under-correction ${ }^{38-40)}$. Because the segmental PS construct provides a greater three-dimensional vertebral controllability, several reports of surgical outcomes have demonstrated that a better thoracic curve correction with an appropriate LIV selection results in less postoperative coronal decompensation after STF for this curve type. Thus, the segmental PS construct achieves a better instrumented thoracic curve correction and SLCC with lesser correction loss than HRI or $\mathrm{CDI}^{25-28,41,44,47,48,64)}$.

The effect of the approach on SLCC outcomes has also been investigated. Several authors have reported that better SLCC was achieved using anterior STF than using posterior STF with a hook construct, and these studies have speculated that this consequence resulted from surgeons' intentional undercorrection to avoid postoperative coronal decom- 
Table 3. Reported Cobb Measurements and Correction Rates in Patients with STF for King-Moe II/Lenke 1C Curve.

\begin{tabular}{|c|c|c|c|c|c|c|c|c|c|c|c|c|c|}
\hline \multirow[b]{2}{*}{ Author (Year) } & \multirow{2}{*}{$\begin{array}{l}\text { Mean } \\
\text { (Min.) } \\
\text { follow-up } \\
\text { (Year) }\end{array}$} & \multirow[b]{2}{*}{ Curve type } & \multirow[b]{2}{*}{ LIV } & \multirow[b]{2}{*}{$\begin{array}{l}\text { Approaches or } \\
\text { constructs }\end{array}$} & \multirow[b]{2}{*}{$\mathrm{N}$} & \multicolumn{4}{|c|}{ Thoracic curve $\left(^{\circ}\right)$} & \multicolumn{4}{|c|}{ Lumbar curve $\left({ }^{\circ}\right)$} \\
\hline & & & & & & $\begin{array}{l}\text { Pre- } \\
\text { op. }\end{array}$ & $\begin{array}{l}\text { Flex. } \\
(\%)\end{array}$ & Final & $\begin{array}{l}\mathrm{CR} \\
(\%)\end{array}$ & $\begin{array}{l}\text { Pre- } \\
\text { op. }\end{array}$ & $\begin{array}{l}\text { Flex. } \\
(\%)\end{array}$ & Final & $\begin{array}{l}\text { CR } \\
(\%)\end{array}$ \\
\hline Shufflebarger (1990) & $2.4(2)$ & KM II & $\geq \mathrm{L} 2$ & CDI & 34 & NA & NA & NA & $66^{\#}$ & NA & NA & NA & $60^{\#}$ \\
\hline Kalen (1990) & NA (NA) & KM II & NA & HRI, LR, etc. & 58 & 52 & 33 & 39 & 25 & 32 & 75 & 22 & 31 \\
\hline Bridwell (1991) & $1.9^{\#}(1)$ & KM II & NA & CDI & 31 & $53.1^{\#}$ & NA & $26.3^{\#}$ & $51.2^{\#}$ & $38.1^{\#}$ & NA & $24.5^{\#}$ & $35.3^{\#}$ \\
\hline Knapp (1992) & $3.5(2)$ & KM II & SV & HRI & 17 & 58.9 & 42.4 & 37.7 & 37.1 & 41.1 & 61.4 & 27.6 & 34.6 \\
\hline McCall (1992) & $1.6(0.92)$ & KM II & $\geq \mathrm{L} 1$ & CDI & 23 & $53.3^{\#}$ & $47.7^{\#}$ & NA & NA & $40.9^{\#}$ & $87.3^{\#}$ & NA & NA \\
\hline Lenke (1992) & $2.7(0.25)$ & KM II & $\geq \mathrm{L} 1$ & CDI & 27 & 61 & 44 & 35 & 40 & 44 & 70 & 28 & 36 \\
\hline Richards (1992) & $2(0.5)$ & KM II & $\geq \mathrm{L} 2$ & CDI, TSRH & 24 & 61 & 36 & 32 & $48^{\#}$ & 49 & 73 & 36 & $27^{\#}$ \\
\hline Benli (1996) & $4.1(2)$ & KM II & NA & CDI & 12 & 74.5 & NA & $56.7^{\#}$ & $24^{\#}$ & 42.2 & NA & $25.6^{\#}$ & $39^{\#}$ \\
\hline McCance (1998) & $5.5(2)$ & KM II & $\geq \mathrm{L} 1$ & HRI, HRI+LR, Hook & 67 & $56.3^{\#}$ & $44.9^{\#}$ & $34.1^{\#}$ & $39.4^{\#}$ & $44.3^{\#}$ & $78.1^{\#}$ & $31.3^{\#}$ & $29.3^{\#}$ \\
\hline \multirow[t]{2}{*}{ Lenke (1999) } & $2(2)$ & MTLC & $\geq \mathrm{L} 1$ & PSF (Hook) & 10 & 67 & $40^{\#}$ & 49 & 27 & 53 & $66^{\#}$ & 37 & 30 \\
\hline & & & & ASF & 7 & 65 & $34^{\#}$ & 27 & 59 & 42 & $71^{\#}$ & 21 & 50 \\
\hline Burton (1999) & $4.8(3.7)$ & KM IIA & $\mathrm{T} 12 *$ & Hybrid (ISOLA) & 6 & 63 & NA & 31 & 51 & 42 & NA & 28 & 33 \\
\hline Frez (2000) & $4(3)$ & KM II & $\geq \mathrm{L} 1$ & HRI+LR+SPW & 24 & 60.2 & 37 & $43.4^{\#}$ & $28^{\#}$ & 40.7 & $67^{\#}$ & $32.2^{\#}$ & $21^{\#}$ \\
\hline van Rhijn (2002) & $6(2)$ & KM II & $\geq \mathrm{L} 2$ & HRI+SLW & 27 & 54 & $46^{\#}$ & 31 & 43 & 34 & $85^{\#}$ & 22 & 35 \\
\hline \multirow[t]{2}{*}{ Dobbs (2004) } & NA (2) & Lenke 12/C & $\geq \mathrm{L} 1$ & PSF (Hook) & 19 & 62.2 & $43^{\#}$ & 37.5 & 40 & 44.4 & $71^{\#}$ & 28.8 & 36 \\
\hline & & & & ASF & 16 & 62.2 & $38^{\#}$ & 33.2 & 47 & 44.8 & $73^{\#}$ & 26.8 & 41 \\
\hline \multirow[t]{2}{*}{ Edwards (2004) } & $5(2)$ & Lenke 12/C & $\geq \mathrm{L} 1$ & PSF (Hook+Wire/PS) & 26 & 62 & NA & 42 & 32 & 48 & NA & 32 & 33 \\
\hline & & & & ASF & 15 & 56 & NA & 32 & 43 & 44 & NA & 27 & 39 \\
\hline Suk (2005) & NA (5) & KM II & NA & PS & 122 & 50 & 51 & 16 & 69 & 33 & 114 & 12 & 62 \\
\hline \multirow[t]{2}{*}{ Dobbs (2006) } & $3(2)$ & MTLC & $\geq \mathrm{L} 1$ & PS & 34 & 62 & 52 & 29.4 & 53 & 44.6 & 46 & 28.4 & $36^{\#}$ \\
\hline & $4(2)$ & & & Hook & 32 & 61 & 53 & 41.1 & 34 & 49.5 & 49 & 37.5 & $24^{\#}$ \\
\hline Chang (2007) & $3.5(2)$ & Lenke 12/C & $\geq \mathrm{L} 1$ & PS & 37 & 63 & 35 & 11 & 83 & 47 & 66 & 9 & 81 \\
\hline \multirow[t]{2}{*}{ Patel (2008) } & $2(2)$ & Lenke1234/ & $\geq \mathrm{L} 1$ & PSF (Hook, Hybrid) & 44 & 57 & 44 & 26 & 54 & 40 & 67 & 21 & 49 \\
\hline & & $\mathrm{BC}$ & & ASF & 132 & 53 & 46 & 24 & 55 & 39 & 73 & 22 & 44 \\
\hline Chang (2010) & $6.8(5)$ & MTLC & $\mathrm{T} 12, \mathrm{~L} 1$ & PSF, ASF & 32 & 61.6 & 41 & 39.8 & 36 & 47.2 & 61 & 33.2 & 25.3 \\
\hline Abel (2011) & $3(2)$ & Lenke 13/BC & $\geq$ L Apex & PSF & 123 & 58.9 & NA & 26.7 & $55^{\#}$ & 42.2 & NA & 22.2 & $47^{\#}$ \\
\hline \multirow[t]{3}{*}{ Takahashi (2011) } & $2(2)$ & Lenke & $\geq \mathrm{L} 1$ & PSF (PS, Hook, SBE & 93 & 54 & 50 & $23^{\#}$ & 58 & 36 & 78 & $19^{\#}$ & 46 \\
\hline & & & & Hybrid), ASF SAE & 66 & 51 & 49 & $23^{\#}$ & 54 & 39 & 73 & $23^{\#}$ & 42 \\
\hline & & & & EBS & 13 & 50 & 57 & $21^{\#}$ & 58 & 39 & 72 & $23^{\#}$ & 42 \\
\hline Wang (2012) & $2(2)$ & Lenke 1C & $\geq \mathrm{L} 1$ & PS & 44 & $54.4^{\#}$ & $39.4^{\#}$ & $21.8^{\#}$ & $61^{\#}$ & $41.7^{\#}$ & $75.1^{\#}$ & $24.2^{\#}$ & $42.5^{\#}$ \\
\hline Wang (2012) & $2(2)$ & Lenke 1C & $\geq \mathrm{L} 1$ & PS & 29 & 55.3 & NA & 22 & 61 & 43 & NA & 25.3 & 41 \\
\hline Larson (2012) & $20.7(17)$ & $\begin{array}{l}\text { Lenke } \\
1 \mathrm{BC} / 3 \mathrm{C}\end{array}$ & $\geq \mathrm{L} 2$ & CDI, TSRH & 14 & 60 & NA & 31 & $48^{\#}$ & 44 & 86 & 27 & 38 \\
\hline Yong (2012) & $2(2)$ & Lenke 1C & $\geq \mathrm{T} 12$ & Thoracoscopic ASF & 24 & 53 & $59^{\#}$ & 24.9 & 52.5 & 43.5 & $74^{\#}$ & 25.4 & 41.8 \\
\hline Ilgenfritz (2013) & $5(5)$ & Lenke 1C & $\geq \mathrm{L} 1$ & ASF, PSF & 24 & 49 & NA & 26.5 & 46 & 40 & NA & 24.4 & 39 \\
\hline Demura (2013) & NA (2) & Lenke 1C & $>\mathrm{L} 1$ & $\begin{array}{c}\text { PSF (PS, Hybrid), } \\
\text { ASF }\end{array}$ & 53 & 49.3 & $45^{\#}$ & 22.3 & 54.3 & 39 & $69^{\#}$ & 23.1 & 40.4 \\
\hline Liljenqvist (2013) & $4(2)$ & Lenke $12 / \mathrm{C}$ & $\geq \mathrm{L} 1$ & ASF (open) & 28 & 61.6 & 42.9 & 29.3 & $52^{\#}$ & 47.7 & 66.2 & 30.5 & $36.1^{\#}$ \\
\hline Chang (2014) & NA (2) & Lenke $12 / \mathrm{C}$ & $\geq \mathrm{L} 1$ & PS & 150 & 65 & NA & 18 & 72 & 51 & NA & 18 & 65 \\
\hline Schulz (2014) & NA (2) & Lenke $1234 / \mathrm{C}$ & $\geq \mathrm{L} 1$ & NA & 106 & 53 & 40 & 24 & 54 & 41 & 64 & 25 & 40 \\
\hline Enercan (2015) & $11.4(10)$ & Lenke 1/BC & $\mathrm{L} 1$ & PS & 25 & 58 & 43 & 17 & $71^{\#}$ & 41 & 63 & 13 & $68^{\#}$ \\
\hline Celestre (2015) & $2(2)$ & Lenke 1C & $\geq \mathrm{L} 1$ & PSF & 38 & 58.9 & NA & 28.7 & $51.3^{\#}$ & 45.8 & NA & 25.5 & $44.3^{\#}$ \\
\hline Skaggs (2016) & $3.7(2)$ & Lenke $1234 / \mathrm{C}$ & $\geq \mathrm{L} 1$ & PS & 33 & $60.4^{\#}$ & $46.7^{\#}$ & $25.2^{\#}$ & $57.5^{\#}$ & $46.5^{\#}$ & $59.6^{\#}$ & $26.8^{\#}$ & $42.3^{\#}$ \\
\hline Sullivan (2017) & $2(2)$ & Lenke 1234/C & $\geq \mathrm{L} 1$ & PSF & 121 & 53 & NA & $23^{\#}$ & $56^{\#}$ & 41 & NA & $23^{\#}$ & $43^{\#}$ \\
\hline
\end{tabular}

STF, selective thoracic fusion; Min., minimum; LIV, lowest instrumented vertebra; Flex., flexibility; CR, correction rate; KM II, King-Moe II; CDI, Cotrel-Dubousset instrumentation; NA, not available; HRI, Harrington rod instrumentation; LR, Luque rod; SV, stable vertebra; TSRH, Texas Scottish Rite Hospital; MTLC, major thoracic-lumbar C modifier curves; PSF, posterior spinal fusion; ASF, anterior spinal fusion; SPW, spinous process wiring; SLW, sublaminar wiring; PS, pedicle screw; L, lumbar; SBE, stable vertebra below end vertebra; SAE, stable vertebra at end vertebra; EBS, end vertebra below stable vertebra; *,

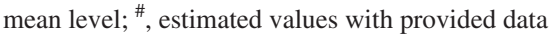

pensation when using the posterior approach ${ }^{38,39)}$. Others have reported that anterior and posterior STF with a hook or hybrid construct have similar SLCC in matched cohorts, with respect to the LIV distribution, thoracic curve correction rate, and preoperative lumbar curve flexibility ${ }^{40,42,67-69)}$.

In most successful cases after STF, the unfused lumbar curve shows significant improvement and accommodates to the corrected thoracic curve, resulting in a balanced spine 

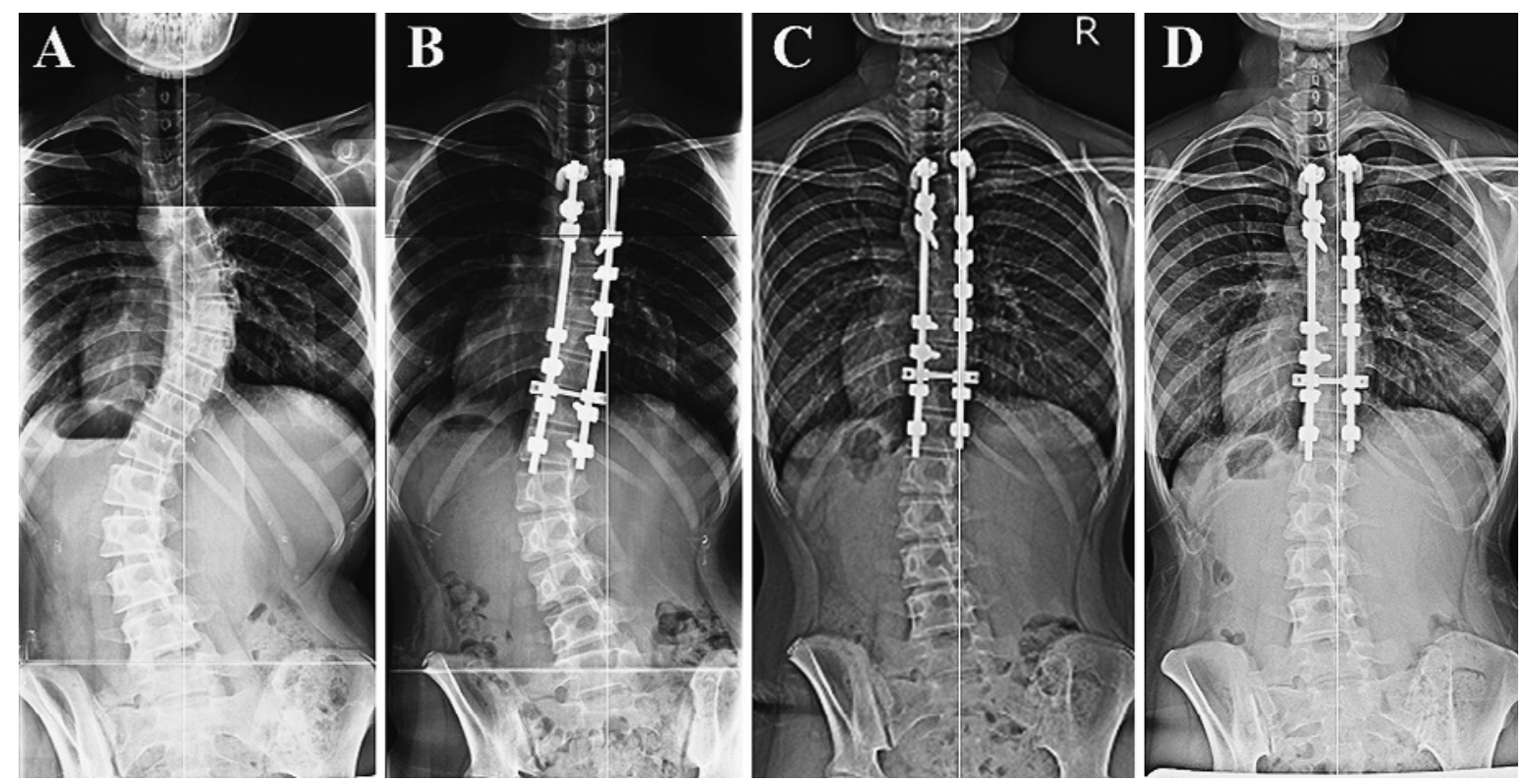

Figure 1. Representative case of a 12-year-old female adolescent idiopathic scoliosis patient with a King-Moe II/ Lenke 1CN curve. (A) Preoperative radiograph. (B) Posterior selective thoracic fusion from T4 to T12. Immediately after surgery, the thoracic curve was corrected from $52^{\circ}$ to $13^{\circ}$ and the lumbar curve was corrected from $44^{\circ}$ to $32^{\circ}$, along with coronal decompensation and trunk shift to the left. (C) While the thoracic curve remained stable thereafter, the lumbar curve continued to spontaneously improve at 6 months after surgery. (D) Remodeling of coronal balance and trunk shift was achieved with successful spontaneous lumbar curve correction $\left(17^{\circ}\right)$ and settling of the lumbosacral curve at 6 years after surgery. (A) and (B), with permission to reprint from Elsevier.

and improved lumbar AVT. However, several studies have found that even with successful postoperative courses, lumbar AVR gains only limited improvement after surgery $^{22,25-27,39,40,60,65,69-73)}$. In contrast, if the unfused lumbar curve cannot accommodate to the corrected thoracic curve, then postoperative coronal decompensation with residual lumbar AVT is likely ${ }^{74-79)}$. A possible explanation for this phenomenon, excluding the postoperative curve progression, is that most SLCC occurs above the lumbar apex immediately after surgery because L4 obliquity and lumbosacral curve persist after surgery ${ }^{19,39,75-78)}$. With continued persistence, postoperative coronal decompensation also remains. In successful cases, immediate postoperative coronal imbalance can be remodeled through the slight loss of lumbar curve correction above the lumbar apex and/or settling in the lumbosacral curve due to potential compensation existing in the relatively flexible lumbar curve ${ }^{19,45,48)}$. Moreover, SLCC is sometimes dynamic, and the unfused lumbar curve continues to improve slightly from immediately after surgery through the final follow-up ${ }^{22,25,26,38,40)}$ (Fig. 1).

Several factors affecting SLCC have been identified, including the preoperative magnitude and flexibility of the lumbar curve, correction of the thoracic curve or LIV tilt, and LIV selection ${ }^{25,29,38,40,42,43,47,56,57,65,66,70,80,81}$. While still controversial, evidence suggests that better SLCC is associated with better thoracic curve correction and LIV selection distal to the lower end vertebra (EV) of the thoracic curve.

In majority of patients undergoing STF for this curve pattern, both the thoracic and lumbar curves are reported to be stable after a 2-year follow-up period, regardless of the approach or the construct used ${ }^{4,6,22,34,39,58,69)}$. However, skeletal immaturity (Risser grade 0-1; open triradiate cartilage) may cause some curve correction loss in the instrumented thoracic and unfused lumbar curves ${ }^{22,39,82,83}$.

Compared with radiographic outcomes of non-STF, the instrumented thoracic curve correction is comparable or slightly less in STF. However, lumbar curve correction and coronal balance preservation after surgery are significantly inferior in STF at the expense of sparing more lumbar mobile segments ${ }^{31,46,62,73,78,84,85)}$.

\section{Coronal Balance}

In King-Moe type II or Lenke 1C curves, preoperative coronal balance is prone to shift to the left, with an incidence of coronal decompensation of $10 \%-40 \%{ }^{34,46,48,86}$, whereas the incidence of postoperative coronal decompensation after STF is reported to be $2 \%-75 \%$ (Table 4). Although the reported rates of revision surgery for treating postoperative coronal decompensation are very low (Table 4), postoperative coronal imbalance is associated with poor patientreported outcomes in pain, self-image, function, and satisfaction, as measured on the Scoliosis Research Society questionnaire, indicating its clinical relevance ${ }^{22,29,39,87}$.

Several causative factors for coronal decompensation after STF have been reported to date (Table 4) and fall under the categories of intrinsic characteristics of spinal deformities, surgical techniques, and LIV selection. Characteristics of 
Table 4. Reported Causative Factors, Incidence, and Revision Cases for Postoperative Coronal Decompensation or Trunk Shift after STF for King-Moe II/Lenke 1C Curve.

\begin{tabular}{|c|c|c|c|c|c|c|}
\hline Author (Year) & $\begin{array}{l}\text { Approaches or } \\
\text { constructs }\end{array}$ & $\begin{array}{l}\text { CD or } \\
\text { TS }\end{array}$ & Curve type & Causative factors & $\begin{array}{l}\text { Incidence }(\%) \\
(\mathrm{N})\end{array}$ & $\begin{array}{c}\text { Revision } \\
\text { cases }\end{array}$ \\
\hline $\begin{array}{l}\text { Schufflebarger } \\
\text { (1990) }\end{array}$ & CDI & $\mathrm{CD}$ & King-Moe II & hook pattern & NA & NA \\
\hline $\begin{array}{l}\text { Thompson } \\
\text { (1990) }\end{array}$ & $\mathrm{CDI}$ & $\mathrm{CD}$ & King-Moe II & $\begin{array}{l}\text { LIV selection, derotation, overcor- } \\
\text { rection }\end{array}$ & $75(9 / 12)$ & NA \\
\hline Mason (1991) & HRI, CDI & $\mathrm{TS}$ & PTCL & $\begin{array}{c}\text { lumbosacral angle }>15^{\circ}, \text { L4 tilt }>12^{\circ}, \\
\text { L-AVT }>2 \mathrm{~cm}\end{array}$ & $\begin{array}{l}\text { HRI; } 4 \text { (1/24), } \\
\text { CDI; } 41 \text { (7/17) }\end{array}$ & NA \\
\hline Moore (1991) & CDI & $\mathrm{CD}$ & King-Moe II & LIV selection, derotaion & NA & NA \\
\hline $\begin{array}{l}\text { Bridwell } \\
(1991)\end{array}$ & CDI & $\mathrm{CD}$ & King-Moe II & $\begin{array}{l}\text { derotation, overcorrection, hook } \\
\text { pattern, rod contour }\end{array}$ & $29(9 / 31)$ & 3 \\
\hline Lenke (1992) & CDI & $\mathrm{CD}$ & King-Moe II & smaller T/L ratio, derotation & $16(3 / 19)$ & 3 \\
\hline McCall (1992) & $\mathrm{CDI}$ & $\mathrm{CD}$ & King-Moe II & L Cobb $>45^{\circ}$, F.I. $<25$, overcorrection & $17(4 / 23)$ & NA \\
\hline $\begin{array}{l}\text { Richards } \\
\text { (1992) }\end{array}$ & CDI, TSRH & TS & King-Moe II & L Cobb $>40^{\circ}$, persistant L4 obliquity & NA & NA \\
\hline King (1994) & HRI, CDI, ISOLA & $\mathrm{CD}$ & King-Moe II & LIV selection, derotation & NA & NA \\
\hline Benli (1996) & $\mathrm{CDI}$ & $\mathrm{CD}$ & King-Moe II & L Cobb $>40^{\circ}$, overcorrection & NA & NA \\
\hline $\begin{array}{l}\text { McCance } \\
\text { (1998) }\end{array}$ & $\begin{array}{l}\text { HRI, HRI+Luque, } \\
\text { Hook }\end{array}$ & $\mathrm{CD}$ & King-Moe II & LIV selection, large Cobb $(\mathrm{T}, \mathrm{L})$ & $30(20 / 67)$ & 0 \\
\hline $\begin{array}{l}\text { Edwards } \\
(2004)\end{array}$ & $\begin{array}{c}\text { PSF (Hook+Wire/PS), } \\
\text { ASF }\end{array}$ & $\mathrm{CD}$ & Lenke $1 \mathrm{C}, 2 \mathrm{C}$ & preop. CD & $\begin{array}{l}\text { PSF; } 46(12 / 26) \\
\text { ASF; } 53(8 / 15)\end{array}$ & 0 \\
\hline Dobbs (2004) & PSF (Hook), ASF & $\mathrm{CD}$ & $\begin{array}{l}\text { Lenke } 1 \mathrm{BC} \\
2 \mathrm{BC}\end{array}$ & preop. $\mathrm{CD}$, overcorrection & $\begin{array}{l}\text { PSF; } 9(4 / 44) \\
\text { ASF; } 2(1 / 56)\end{array}$ & 0 \\
\hline Suk (2005) & PS & $\mathrm{CD}$ & King-Moe II & overcorrection & $6(7 / 122)$ & 0 \\
\hline Dobbs (2006) & PSF (Hook, PS) & $\mathrm{CD}$ & $\begin{array}{l}\text { PTCL (lumbar } \\
\text { C modifier) }\end{array}$ & NA & $\begin{array}{l}\text { Hook; } 41(13 / 32) \\
\quad \text { PS; } 12(4 / 34)\end{array}$ & 0 \\
\hline Wang (2012) & PS & TS & Lenke 1C & LIV selection, smaller $\mathrm{T} / \mathrm{L}$ ratio & NA & NA \\
\hline Demura (2013) & $\begin{array}{c}\text { PSF (PS, Hybrid), } \\
\text { ASF }\end{array}$ & $\mathrm{CD}$ & Lenke 1C & preop. $\mathrm{CD}$ & $42(22 / 53)$ & NA \\
\hline Chang (2014) & PS & $\mathrm{CD}$ & Lenke $1234 \mathrm{C}$ & NA & $5(8 / 148)$ & 0 \\
\hline $\begin{array}{l}\text { Ishikawa } \\
\text { (2017) }\end{array}$ & PS & $\mathrm{CD}$ & Lenke $1 \mathrm{C}, 2 \mathrm{C}$ & $\begin{array}{l}\text { LIV selection, preop. coronal } \\
\text { imbalance to the left }\end{array}$ & $14(3 / 21)$ & 0 \\
\hline Sullivan (2017) & PSF & $\mathrm{CD}$ & Lenke $1234 \mathrm{C}$ & preop. $\mathrm{CD}$ & $41(49 / 121)$ & NA \\
\hline
\end{tabular}

STF, selective thoracic fusion; CD, coronal decompensation; TS, trunk shift; CDI, Cotrel-Dubousset instrumentation; NA, not available; LIV, lowest instrumented vertebra; HRI, Harrington rod instrumentation; PTCL, primary thoracic and compensatory lumbar curves; L, lumbar; AVT, apical vertebral translation; T, thoracic; F.I., flexibility index; TSRH, Texas Scottish Rite Hospital; PSF, posterior spinal fusion; ASF, anterior spinal fusion; PS, pedicle screw

spinal deformities include preoperative coronal decompensation to the left; larger and stiffer lumbar curve; smaller flexibility index; smaller ratios of the thoracic curve to lumbar curve in the Cobb angle, AVT, and AVR; and persistent lumbosacral curve. Surgical techniques associated with postoperative coronal decompensation include thoracic curve overcorrection (to the point where the correction is greater than the preoperative flexibility on side-bending), derotation maneuver, hook pattern at LIV, and rod contour.

In the HRI era, postoperative coronal decompensation was not a significant problem in cases with LIV at the SV and $\mathrm{NV}$, as proposed by King and Moe. However, postoperative coronal decompensation became a significant problem in the CDI era, even in cases following the King-Moe's rule on LIV selection ${ }^{18,19,35,37,88,89)}$.

Thoracic curve overcorrection using a derotation maneuver, larger lumbar curve, or LIV selection was a major causative factor in the CDI era ${ }^{19,20,33-37,61,70,74,75,88,90-92)}$. In contrast, a large thoracic curve correction with appropriate LIV selection is less likely to result in postoperative coronal decom- pensation in the PS construct than in the hook construct. This discrepancy may result from different mechanisms whereby corrective forces are applied between the hook and PS constructs. During the derotation maneuver in the posterior approach, the derotation force applied to the thoracic curve is transmitted to the lumbar curve less with the PS than with the hook construct. This difference is because PSs at the lower foundation can be used to regulate the aggravation of the lumbar curve and may even somewhat correct the uppermost portion of the lumbar curve by the derotation of LIV in the direction opposite of the thoracic curve derotation $^{25,41,93,94)}$. Therefore, coronal decompensation is less likely after surgery using the PS construct, even when a better thoracic curve correction is achieved.

To avoid postoperative coronal decompensation using CDI, recommendations include the use of a compression mode hook instead of a distraction mode hook at the thoracolumbar junction on the thoracic concave side and the use of a reverse-bent rod. Otherwise, the distraction force imparted to the convex side of the lumbar curve aggravates the 

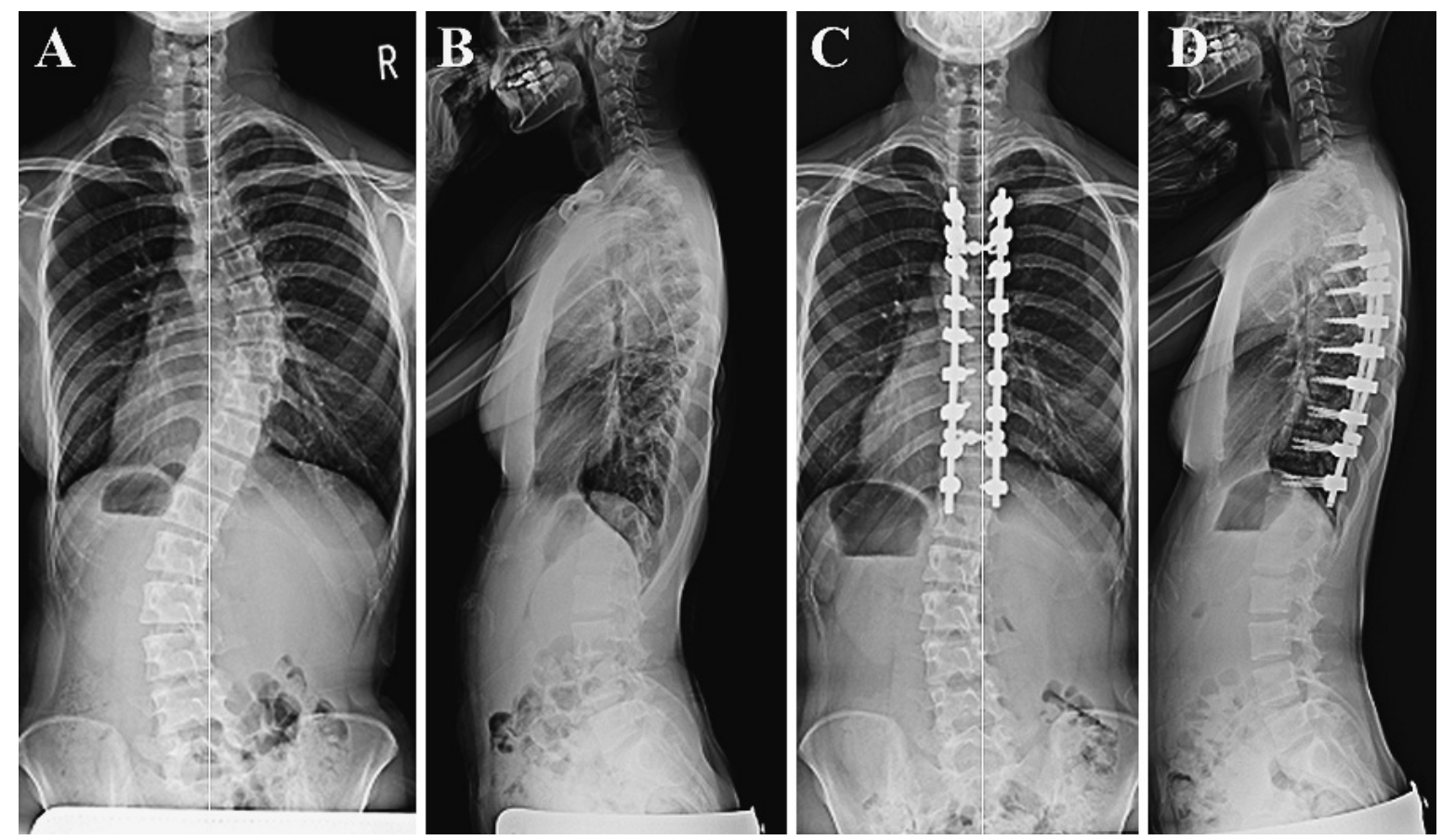

Figure 2. Representative case of an 18-year-old female adolescent idiopathic scoliosis patient with a King-Moe II/ Lenke 1CN curve. (A) Preoperative postero-anterior radiograph. (B) Preoperative lateral radiograph. (C) Posterior selective thoracic fusion with all-pedicle screw construct from T4 to T12 (stable vertebra). Postoperative postero-anterior radiograph taken at 6 weeks after surgery already shows acceptable spontaneous lumbar curve correction and coronal balance. (D) Postoperative lateral radiograph shows normal sagittal alignment.

lumbar curve, resulting in coronal and sagittal decompensa$\operatorname{tion}^{35-38)}$.

Preoperative coronal decompensation to the left has been consistently reported as a major causative factor in postoperative coronal decompensation for all constructs ${ }^{39,40,46-48,54}$. The reported rate of postoperative coronal decompensation after STF for patients with preoperative coronal imbalance to the left ranges from $41 \%$ to $57 \%$, a rate higher than that for patients who were preoperatively balanced $(31 \%)^{46,54)}$. The reason for this difference may be that lumbar curves with a low compensatory capacity to maintain coronal balance or persistent lumbosacral curve cannot accommodate to either the preoperative primary thoracic curve or the instrumented and corrected thoracic curve. This possibility is supported by findings that a better SLCC results in less postoperative coronal decompensation after $\mathrm{STF}^{25,41,43,54,75,76)}$.

Less attention has been paid to the persistent lumbosacral curve, or L4 tilt, and sacral obliquity as causative factors for postoperative coronal decompensation. Mason and Carango speculate that a greater preoperative lumbosacral angle (> $15^{\circ}$ ) is a causative factor of postoperative coronal decompensation because SLCC mostly occurs between LIV and lumbar apex and less often below the lumbar apex ${ }^{19,75,76,78,80)}$.

Larger $\left(>40^{\circ}-45^{\circ}\right)$ and stiffer lumbar curves with a low flexibility index $(<25)$ are considered more prone to postoperative coronal decompensation ${ }^{10,19,20,34,46,62,88,90)}$. However, recent reports using the PS construct have demonstrated that larger and stiffer lumbar curves, including some of Lenke 3C and 4C curves, successfully responded to the instru- mented thoracic curve without an increased rate of postoperative coronal decompensation ${ }^{26,28,95,96)}$.

Smaller ratios (close to 1.0) of the thoracic curve to the lumbar curve in the Cobb angle, AVT, and AVR have been identified as causative factors for postoperative coronal decompensation with $\mathrm{CDI}^{18,88)}$. Using a cut-off value of 1.2 for this ratio may not necessarily guarantee successful postoperative outcomes on coronal balance or lumbar curve, as shown using a recent PS construct ${ }^{26,28,29)}$. However, patients with a greater difference in size, particularly in the AVT between the thoracic and lumbar curves, are still considered to be more safely treated with $\mathrm{STF}^{22,42,44,75)}$.

While conflicting recommendations are reported for LIV selection, most surgeons prefer LIV at SV for cases in which SV is located at or distal to the lower EV of the thoracic curve because fixation distal to $\mathrm{SV}$ results in postoperative coronal decompensation, whereas fusing short of EV causes postoperative curve progression ${ }^{17,18,25,33,34,38,43,44,47,48,78,87)}$. In the CDI era, several authors reported that STF fused to SV resulted in postoperative coronal decompensation in some cases and thus, recommended shorter fusion levels $\mathrm{s}^{35,37,61,75,97)}$. Conversely, coronal balance seems to be well maintained after surgery if LIV is placed near SV (within one level) using a recent PS construct (Fig. 2). If LIV is placed at or just above the lumbar apex, then coronal balance often shifts to the left with decompensation due to the persistent lumbosacral curve $\mathrm{s}^{45,47,48,63,76,78}$. In patients with preoperative severe coronal decompensation to the left, SV is located near the thoracic apex and above the lower $\mathrm{EV}$ of thoracic curve. In 

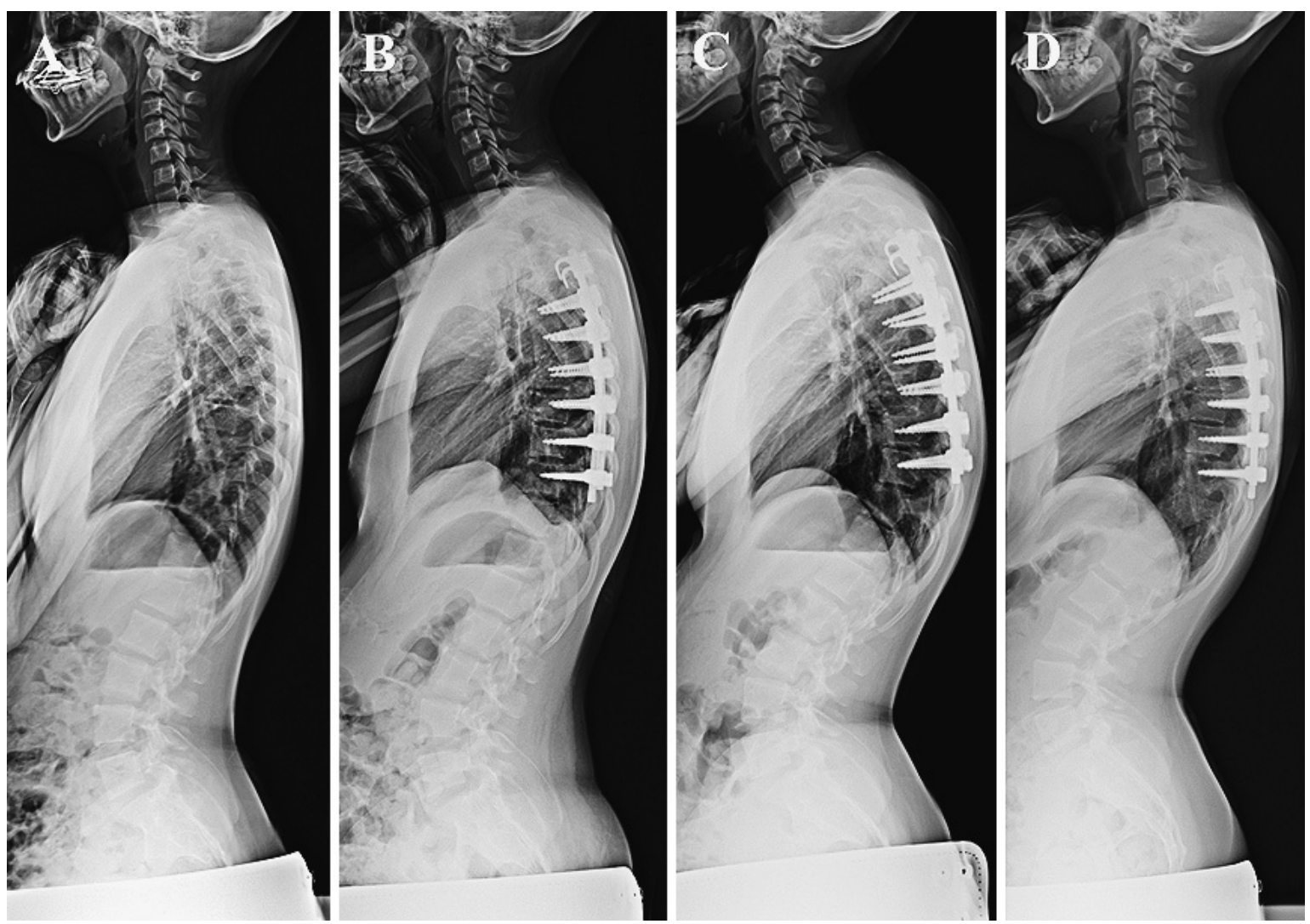

Figure 3. Representative case of a 15-year-old female adolescent idiopathic scoliosis patient with a King-Moe II/ Lenke 1CN curve. (A) Preoperative lateral radiograph. (B) Posterior selective thoracic fusion from T4 to T10. Preoperative thoracolumbar kyphosis of $15^{\circ}$ was corrected to $18^{\circ}$ immediately after surgery. (C) Thoracolumbar kyphosis subsequently increased to $26^{\circ}$ at 3 months after surgery. (D) The increased postoperative thoracolumbar kyphosis remains stable and has been compensated by increased lumbar lordosis thus far, as seen on the radiograph taken at 1.5 years after surgery.

such cases, LIV should be extended at least to EV to avoid postoperative curve progression, or may need to be placed beyond the lumbar apex (non-STF) because the ability of STF to maintain coronal balance after surgery may be limited $^{43,454-48,54,87,98)}$

Remodeling of the trunk shift or coronal imbalance, which may be attributed to postural reflex, is reported to occur in some patients after $\operatorname{STF}^{37,45,48,58,68,79,9,87,99)}$ (Fig. 1). As with coronal curve correction, coronal balance usually remains stable after 2 years post surgery ${ }^{6,7,22,34,39,69)}$.

\section{Thoracolumbar Kyphosis}

Sagittal malalignment with a significant positive sagittal imbalance is the most significant factor influencing the patient's health status measures on back pain and function in adulthood $^{100}$. Therefore, achieving a normal sagittal alignment with normal thoracolumbar kyphosis after STF is mandatory and should override optimizing postoperative coronal curvature.

Reported causative factors for postoperative distal junctional kyphosis include LIV at the sagittal thoracolumbar apex, distraction mode hook placement at the thoracolumbar junction with CDI, and a significant preoperative thora- columbar kyphosis ${ }^{22,24,3,133,36,37,37,3988}$. Although mild thoracolumbar kyphosis after STF is usually well tolerated during young adulthood with compensatory capacity to maintain the sagittal balance ${ }^{83.88}$ (Fig. 3), follow-up studies of patients aged $>50$ years with decreased compensatory capacity are needed to document its long-term effects on radiographic global sagittal alignment and balance and clinical outcomes.

As shown in Table 5, thoracolumbar kyphosis is maintained or slightly increases (kyphotic) after STF and decreases (lordotic) after non-STF surgery.

\section{Conclusion}

Although STF has been validated in general for the treatment of King-Moe type II or Lenke 1C curve in AIS, controversies still remain regarding surgical indications and outcomes.

Careful preoperative evaluations of the clinical appearance, radiographic parameters, and patient's expectations of the postoperative course and appropriate surgical techniques are required to achieve successful surgical outcomes for this complex spinal deformity.

Long-term impacts of residual lumbar curve, coronal decompensation, and mild thoracolumbar kyphosis on clinical 
Table 5. Reported Thoracolumbar Kyphosis in Patients Treated with STF or Non-STF for Lenke 1C Curve.

\begin{tabular}{|c|c|c|c|c|c|c|c|c|c|}
\hline \multirow{3}{*}{ Author (Year) } & \multirow{3}{*}{ Curve type } & \multirow{3}{*}{$\begin{array}{l}\text { Approaches or } \\
\text { constructs }\end{array}$} & \multirow{3}{*}{$\begin{array}{l}\text { LIV for } \\
\text { STF }\end{array}$} & \multicolumn{6}{|c|}{ Thoracolumbar kyphosis (T10-L2) } \\
\hline & & & & \multicolumn{3}{|c|}{ STF } & \multicolumn{3}{|c|}{ non-STF } \\
\hline & & & & $\mathrm{N}$ & Preop. $\left({ }^{\circ}\right)$ & Final $\left({ }^{\circ}\right)$ & $\mathrm{N}$ & Preop. $\left({ }^{\circ}\right)$ & Final $\left({ }^{\circ}\right)$ \\
\hline Newton (2003) & Lenke $1 \mathrm{~B}, 1 \mathrm{C}$ & ASF, PSF & $\geq \mathrm{L} 1$ & 168 & -3 & - & 35 & -2 & - \\
\hline \multirow[t]{2}{*}{ Edwards (2004) } & Lenke 1C, 2C & PSF (Hook+Wire/PS) & $\geq \mathrm{L} 1$ & 26 & -6 & -1 & 0 & - & - \\
\hline & & ASF & & 15 & -3 & -3 & 0 & - & - \\
\hline Chang (2007) & Lenke 1C, 2C & PS & $\geq \mathrm{L} 1$ & 37 & -2 & -5 & 0 & - & - \\
\hline Abel (2011) & Lenke $1 \mathrm{BC}, 3 \mathrm{BC}$ & PSF & $\geq$ L-Apex & 123 & -2.3 & 2.7 & 81 & -1.1 & -7.5 \\
\hline Crawford (2013) & Lenke 1C & PSF & $\geq \mathrm{L} 1$ & 138 & -2.1 & - & 126 & -0.3 & - \\
\hline Celestre (2015) & Lenke 1C & PSF & $\geq \mathrm{L} 1$ & 38 & -1.5 & 4.3 & 38 & 1.8 & -9 \\
\hline
\end{tabular}

STF, selective thoracic fusion; LIV, lowest instrumented vertebra; ASF, anterior spinal fusion; PSF, posterior spinal fusion; PS, pedicle screw; L, lumbar

outcomes after STF, along with optimal indications and strategy for STF, should further be assessed.

Conflicts of Interest: The authors declare that there are no relevant conflicts of interest.

Acknowledgement: The authors would like to thank Enago (www.enago.jp) for the English language review.

Author Contributions: Masayuki Ishikawa wrote and prepared the manuscript, and all of the authors participated in the study design. All authors have read, reviewed, and approved the article.

\section{References}

1. Nachemson A. A long term follow-up study of non-treated scoliosis. Acta Orthop Scand. 1968;39(4):466-76.

2. Weinstein SL, Zavala DC, Ponseti IV. Idiopathic scoliosis: longterm follow-up and prognosis in untreated patients. J Bone Joint Surg Am. 1981;63(5):702-12.

3. Weinstein SL, Ponseti IV. Curve progression in idiopathic scoliosis. J Bone Joint Surg Am. 1983;65(4):447-55.

4. Edgar MA, Mehta MH. Long-term follow-up of fused and unfused idiopathic scoliosis. J Bone Joint Surg Br. 1988;70(5):7126.

5. Dickson JH, Erwin WD, Rossi D. Harrington instrumentation and arthrodesis for idiopathic scoliosis. A twenty-one-year follow-up. J Bone Joint Surg Am. 1990;72(5):678-83.

6. Larson AN, Fletcher ND, Daniel C, et al. Lumbar curve is stable after selective thoracic fusion for adolescent idiopathic scoliosis: a 20-year follow-up. Spine. 2012;37(10):833-9.

7. Enercan M, Kahraman S, Cobanoglu M, et al. Selective thoracic fusion provides similar health-related quality of life but can cause more lumbar disc and facet joint degeneration: a comparison of adolescent idiopathic scoliosis patients with normal population 10 years after surgery. Spine Deform. 2015;3(5):469-75.

8. Iida T, Suzuki N, Kono K, et al. Minimum 20 years long-term clinical outcome after spinal fusion and instrumentation for scoliosis: comparison of the SRS-22 patient questionnaire with that in nonscoliosis group. Spine. 2015;40(16):E922-8.

9. Cochran T, Irstam L, Nachemson A. Long-term anatomic and functional changes in patients with adolescent idiopathic scoliosis treated by Harrington rod fusion. Spine. 1983;8(6):576-84.

10. Large DF, Doig WG, Dickens DR, et al. Surgical treatment of double major scoliosis. Improvement of the lumbar curve after fusion of the thoracic curve. J Bone Joint Surg Br. 1991;73(1): 121-4.

11. Danielsson AJ, Nachemson AL. Back pain and function 23 years after fusion for adolescent idiopathic scoliosis: a case-control study-part II. Spine. 2003;28(18):E373-83.

12. Helenius I, Remes V, Yrjönen T, et al. Harrington and CotrelDubousset instrumentation in adolescent idiopathic scoliosis. Long-term functional and radiographic outcomes. J Bone Joint Surg Am. 2003;85(12):2303-9.

13. Green DW, Lawhorne TW 3rd, Widmann RF, et al. Long-term magnetic resonance imaging follow-up demonstrates minimal transitional level lumbar disc degeneration after posterior spine fusion for adolescent idiopathic scoliosis. Spine. 2011;36(23): 1948-54.

14. Nohara A, Kawakami N, Saito T, et al. Comparison of surgical outcomes between anterior fusion and posterior fusion in patients with AIS Lenke Type 1 or 2 that underwent selective thoracic fusion -long-term follow-up study longer than 10 postoperative years. Spine. 2015;40(21):1681-9.

15. Marks MC, Bastrom TP, Petcharaporn M, et al. The effect of time and fusion length on motion of the unfused lumbar segments in adolescent idiopathic scoliosis. Spine Deform. 2015;3 (6):549-53.

16. Moe JH. A critical analysis of methods of fusion for scoliosis. an evaluation in two hundred and sixty-six patients. J Bone Joint Surg Am. 1958;40(3):529-54.

17. King HA, Moe JH, Bradford DS, et al. The selection of fusion levels in thoracic idiopathic scoliosis. J Bone Joint Surg Am. 1983;65(9):1302-13.

18. Lenke LG, Bridwell KH, Baldus C, et al. Preventing decompensation in King type II curves treated with Cotrel-Dubousset instrumentation. Strict guidelines for selective thoracic fusion. Spine. 1992;17(8S):S274-81.

19. Richards BS. Lumbar curve response in type II idiopathic scoliosis after posterior instrumentation of the thoracic curve. Spine. 1992;17(8S):S282-6.

20. McCall RE, Bronson W. Criteria for selective fusion in idiopathic scoliosis using Cotrel-Dubousset instrumentation. J Pediatr Orthop. 1992;12(4):475-9.

21. Lenke LG, Betz RR, Harms J, et al. Adolescent idiopathic scoliosis: a new classification to determine extent of spinal arthrodesis. J Bone Joint Surg Am. 2001;83(8):1169-81.

22. Lenke LG, Edwards CC 2nd, Bridwell KH. The Lenke classification of adolescent idiopathic scoliosis: how it organizes curve patterns as a template to perform selective fusions of the spine. 
Spine. 2003;28(20S):S199-207.

23. Majd ME, Holt RT, Castro FP. Selection of fusion levels in scoliosis surgery. J Spinal Disord Tech. 2003;16(1):71-82.

24. Qiu G, Zhang J, Wang Y, et al. A new operative classification of idiopathic scoliosis: a peking union medical college method. Spine. 2005;30(12):1419-26.

25. Chang KW, Chang KI, Wu CM. Enhanced capacity for spontaneous correction of lumbar curve in the treatment of major thoracic-compensatory $\mathrm{C}$ modifier lumbar curve pattern in idiopathic scoliosis. Spine. 2007;32(26):3020-9.

26. Chang KW, Leng $X$, Zhao W, et al. Broader curve criteria for selective thoracic fusion. Spine. 2011;36(20):1658-64.

27. Chang KW, Chen YY, Leng X, et al. Guan-Din method: a novel surgical technique for selective thoracic fusion to maximize the rate of selective thoracic fusion and compensatory correction. Spine. 2014;39(4):E284-93.

28. Chang KW, Chen YY, Wu CM, et al. Could structural and noncompensatory Lenke 3 and $4 \mathrm{C}$ lumbar curves be nonstructural and compensatory? Lenke 1,2, 3, and 4 curve types were similar and could be considered collectively as a single indication for selective thoracic fusion. Spine. 2014;39(22):1850-9.

29. Schulz J, Asghar J, Bastrom T, et al. Optimal radiographical criteria after selective thoracic fusion for patients with adolescent idiopathic scoliosis with a C lumbar modifier: does adherence to current guidelines predict success? Spine. 2014;39(23):E136873.

30. Lenke LG, Betz RR, Clements D, et al. Curve prevalence of a new classification of operative adolescent idiopathic scoliosis: does classification correlate with treatment? Spine. 2002;27(6): 604-11.

31. Newton PO, Faro FD, Lenke LG, et al. Factors involved in the decision to perform a selective versus nonselective fusion of Lenke 1B and 1C (King-Moe II) curves in adolescent idiopathic scoliosis. Spine. 2003;28(20S):S217-23.

32. Crawford CH 3rd, Lenke LG, Sucato DJ, et al. Selective thoracic fusion in Lenke 1C curves: prevalence and criteria. Spine. 2013;38(16):1380-5.

33. King HA. Analysis and treatment of type II idiopathic scoliosis. Orthop Clin North Am. 1994;25(2):225-37.

34. McCance SE, Denis F, Lonstein JE, et al. Coronal and sagittal balance in surgically treated adolescent idiopathic scoliosis with the King II curve pattern. A review of 67 consecutive cases having selective thoracic arthrodesis. Spine. 1998;23(19):2063-73.

35. Thompson JP, Transfeldt EE, Bradford DS, et al. Decompensation after Cotrel-Dubousset instrumentation of idiopathic scoliosis. Spine. 1990;15(9):927-31.

36. Shufflebarger HL, Clark CE. Fusion levels and hook patterns in thoracic scoliosis with Cotrel-Dubousset instrumentation. Spine. 1990;15(9):916-20.

37. Bridwell KH, McAllister JW, Betz RR, et al. Coronal decompensation produced by Cotrel-Dubousset "derotation" maneuver for idiopathic right thoracic scoliosis. Spine. 1991;16(7):769-77.

38. Lenke LG, Betz RR, Bridwell KH, et al. Spontaneous lumbar curve coronal correction after selective anterior or posterior thoracic fusion in adolescent idiopathic scoliosis. Spine. 1999;24 (16): 1663-71.

39. Edwards CC 2nd, Lenke LG, Peelle M, et al. Selective thoracic fusion for adolescent idiopathic scoliosis with $\mathrm{C}$ modifier lumbar curves: 2-to 16-year radiographic and clinical results. Spine. 2004;29(5):536-46.

40. Dobbs MB, Lenke LG, Walton T, et al. Can we predict the ultimate lumbar curve in adolescent idiopathic scoliosis patients un- dergoing a selective fusion with undercorrection of the thoracic curve? Spine. 2004;29(3):277-85.

41. Dobbs MB, Lenke LG, Kim YJ, et al. Selective posterior thoracic fusions for adolescent idiopathic scoliosis: comparison of hooks versus pedicle screws. Spine. 2006;31(20):2400-4.

42. Patel PN, Upasani VV, Bastrom TP, et al. Spontaneous lumbar curve correction in selective thoracic fusions of idiopathic scoliosis: a comparison of anterior and posterior approaches. Spine. 2008;33(10):1068-73.

43. Takahashi J, Newton PO, Ugrinow VL, et al. Selective thoracic fusion in adolescent idiopathic scoliosis: factors influencing the selection of the optimal lowest instrumented vertebra. Spine. 2011;36(14):1131-41.

44. Wang Y, Bünger CE, Wu C, et al. Postoperative trunk shift in Lenke 1C scoliosis: what causes it? how can it be prevented? Spine. 2012;37(19):1676-82.

45. Wang $\mathrm{Y}$, Bünger $\mathrm{CE}$, Zhang $\mathrm{Y}$, et al. Postoperative spinal alignment remodeling in Lenke 1C scoliosis treated with selective thoracic fusion. Spine J. 2012;12(1):73-80.

46. Demura S, Yaszay B, Bastrom TP, et al. Is decompensation preoperatively a risk in Lenke 1C curves? Spine. 2013;38(11):E 649-55.

47. Ishikawa M, Cao K, Pang L, et al. Postoperative behavior of thoracolumbar/lumbar curve and coronal balance after posterior thoracic fusion for Lenke $1 \mathrm{C}$ and $2 \mathrm{C}$ adolescent idiopathic scoliosis. J Orthop Sci. 2015;20(1):31-7.

48. Ishikawa M, Cao K, Pang L, et al. Onset and remodeling of coronal imbalance after selective posterior thoracic fusion for Lenke $1 \mathrm{C}$ and $2 \mathrm{C}$ adolescent idiopathic scoliosis (a pilot study). Scoliosis Spinal Disord. 2017;12:16.

49. Harrington PR. Treatment of scoliosis. Correction and internal fixation by spine instrumentation. J Bone Joint Surg Am. 1962; 44(4):591-610.

50. Harrington PR. Technical details in relation to the successful use of instrumentation in scoliosis. Orthop Clin North Am. 1972;3 (1):49-67.

51. Cotrel Y, Dubousset J, Guillaumat M. New universal instrumentation in spinal surgery. Clin Orthop Relat Res. 1988;227:10-23.

52. Lenke LG, Betz RR, Haher TR, et al. Multisurgeon assessment of surgical decision-making in adolescent idiopathic scoliosis: curve classification, operative approach, and fusion levels. Spine. 2001;26(21):2347-53.

53. Newton PO, Upasani VV, Bastrom TP, et al. The deformityflexibility quotient predicts both patient satisfaction and surgeon preference in the treatment of Lenke 1B or 1C curves for adolescent idiopathic scoliosis. Spine. 2009;34(10):1032-9.

54. Sullivan TB, Bastrom TP, Bartley CE, et al. Selective thoracic fusion of a left decompensated main thoracic curve: proceed with caution? Eur Spine J. 2018;27(2):312-8.

55. Sharma $\mathrm{S}$, Andersen $\mathrm{T}$, Wu C, et al. How well do radiologic assessments of truncal and shoulder balance correlate with cosmetic assessment indices in Lenke 1C adolescent idiopathic scoliosis? Clin Spine Surg. 2016;29(8):341-51.

56. Kalen V, Conklin M. The behavior of the unfused lumbar curve following selective thoracic fusion for idiopathic scoliosis. Spine. 1990;15(4):271-4.

57. Knapp DR Jr, Price CT, Jones ET, et al. Choosing fusion levels in progressive thoracic idiopathic scoliosis. Spine. 1992;17(10): 1159-65.

58. Frez R, Cheng JC, Wong EM. Longitudinal changes in trunkal balance after selective fusion of King II curves in adolescent idiopathic scoliosis. Spine. 2000;25(11):1352-9. 
59. van Rhijn LW, Plasmans CM, Veraart BE. No relationship exists between the correction of the thoracic and the lumbar curves after selective thoracic fusion for adolescent idiopathic scoliosis King type II. Eur Spine J. 2002;11(6):550-5.

60. Wood KB, Transfeldt EE, Ogilvie JW, et al. Rotational changes of the vertebral-pelvic axis following Cotrel-Dubousset instrumentation. Spine. 1991;16(8S):S404-8.

61. Moore MR, Baynham GC, Brown CW, et al. Analysis of factors related to truncal decompensation following Cotrel-Dubousset instrumentation. J Spinal Disord. 1991;4(2):188-92.

62. Richards BS, Herring JA, Johnston CE, et al. Treatment of adolescent idiopathic scoliosis using Texas Scottish Rite Hospital instrumentation. Spine. 1994;19(14):1598-605.

63. Burton DC, Asher MA, Lai SM. The selection of fusion levels using torsional correction techniques in the surgical treatment of idiopathic scoliosis. Spine. 1999;24(16):1728-39.

64. Suk SI, Lee SM, Chung ER, et al. Selective thoracic fusion with segmental pedicle screw fixation in the treatment of thoracic idiopathic scoliosis: more than 5-year follow-up. Spine. 2005;30 (14):1602-9.

65. Koller H, Meier O, Albrecht $\mathrm{H}$, et al. Selective thoracic fusion in AIS curves: the definition of target outcomes improves the prediction of spontaneous lumbar curve correction (SLCC). Eur Spine J. 2014;23(6):1263-81.

66. Skaggs DL, Seehausen DA, Yamaguchi KT Jr, et al. Assessment of lowest instrumented vertebra tilt on radiographic measurements in Lenke "C" modifier curves undergoing selective thoracic fusion in adolescent idiopathic scoliosis. Spine Deform. 2016;4(2):125-30.

67. Potter BK, Kuklo TR, Lenke LG. Radiographic outcomes of anterior spinal fusion versus posterior spinal fusion with thoracic pedicle screws for treatment of Lenke type 1 adolescent idiopathic scoliosis curves. Spine. 2005;30(16):1859-66.

68. Yong MR, Izatt MT, Adam CJ, et al. Secondary curve behavior in Lenke type 1C adolescent idiopathic scoliosis after thoracoscopic selective anterior thoracic fusion. Spine. 2012;37(23): 1965-74.

69. Ilgenfritz RM, Yaszay B, Bastrom TP, et al. Lenke $1 \mathrm{C}$ and $5 \mathrm{C}$ spinal deformities fused selectively: 5-year outcomes of the uninstrumented compensatory curves. Spine. 2013;38(8):650-8.

70. Goshi K, Boachie-Adjei O, Moore C, et al. Thoracic scoliosis fusion in adolescent and adult idiopathic scoliosis using posterior translational corrective techniques (Isola): is maximum correction of the thoracic curve detrimental to the unfused lumbar curve? Spine J. 2004;4(2):192-201.

71. Ritzman TF, Upasani VV, Bastrom TP, et al. Comparison of compensatory curve spontaneous derotation after selective thoracic or lumbar fusions in adolescent idiopathic scoliosis. Spine. 2008;33(24):2643-7.

72. Liljenqvist U, Halm H, Bullmann V. Spontaneous lumbar curve correction in selective anterior instrumentation and fusion of idiopathic thoracic scoliosis of Lenke type C. Eur Spine J. 2013;22 (Suppl 2):S138-48.

73. Boniello AJ, Hasan S, Yang S, et al. Selective versus nonselective thoracic fusion in Lenke $1 \mathrm{C}$ curves: a meta-analysis of baseline characteristics and postoperative outcomes. J Neurosurg Spine. 2015;23(6):721-30.

74. Patwardhan AG, Rimkus A, Gavin TM, et al. Geometric analysis of coronal decompensation in idiopathic scoliosis. Spine. 1996; 21(10):1192-200.

75. Mason DE, Carango P. Spinal decompensation in CotrelDubousset instrumentation. Spine. 1991;16(8S):S394-403.
76. Schwender JD, Denis F. Coronal plane imbalance in adolescent idiopathic scoliosis with left lumbar curves exceeding 40 degrees: the role of the lumbosacral hemicurve. Spine. 2000;25 (18):2358-63.

77. Jansen RC, van Rhijn LW, Duinkerke E, et al. Predictability of the spontaneous lumbar curve correction after selective thoracic fusion in idiopathic scoliosis. Eur Spine J. 2007;16(9):1335-42.

78. Abel MF, Herndon SK, Sauer LD, et al. Selective versus nonselective fusion for idiopathic scoliosis: does lumbosacral takeoff angle change? Spine. 2011;36(14):1103-12.

79. Von Lackum WH, Miller JP. Critical observations of the results in the operative treatment of scoliosis. J Bone Joint Surg Am. 1949;31(1):102-6.

80. Mason DE, Schindler A, King N. Estimation of the lumbar curve magnitude with correction of the right thoracic curve in idiopathic scoliosis. J Pediatr Orthop. 1998;18(5):602-5.

81. Imrie M, Yaszay $B$, Bastrom $T P$, et al. Adolescent idiopathic scoliosis: should $100 \%$ correction be the goal? J Pediatr Orthop. 2011;31(1):S9-13.

82. Sponseller PD, Betz R, Newton PO, et al. Differences in curve behavior after fusion in adolescent idiopathic scoliosis patients with open triradiate cartilages. Spine. 2009;34(8):827-31.

83. Bridwell KH. Spine update. Surgical treatment of adolescent idiopathic scoliosis: the basics and the controversies. Spine. 1994; 19(9):1095-100.

84. Puno RM, An KC, Puno RL, et al. Treatment recommendations for idiopathic scoliosis: an assessment of the Lenke classification. Spine. 2003;28(18):2102-14.

85. Celestre PC, Carreon LY, Lenke LG, et al. Sagittal alignment two years after selective and nonselective thoracic fusion for Lenke 1C adolescent idiopathic scoliosis. Spine Deform. 2015;3 (6):560-5.

86. Kwan MK, Chiu CK, Tan PH, et al. Radiological and clinical outcome of selective thoracic fusion (STF) for Lenke 1C and 2C adolescent idiopathic scoliosis (AIS) patients with a minimum follow-up of 2 years. Spine J. 2018 May 4. pii: S1529-9430(18) 30206-7. doi: 10.1016/j.spinee.2018.05.007. [Epub ahead of print]

87. Richards BS. Lenke 1C, King type II curves: surgical recommendations. Orthop Clin North Am. 2007;38(4):511-20.

88. Richards BS, Birch JG, Herring JA, et al. Frontal plane and sagittal plane balance following Cotrel-Dubousset instrumentation for idiopathic scoliosis. Spine. 1989;14(7):733-7.

89. Trobisch PD, Samdani AF, Pahys JM, et al. Postoperative trunk shift in Lenke 1 and 2 curves: how common is it? and analysis of risk factors. Eur Spine J. 2011;20(7):1137-40.

90. Benli IT, Tüzüner M, Akalin S, et al. Spinal imbalance and decompensation problems in patients treated with CotrelDubousset instrumentation. Eur Spine J. 1996;5(6):380-6.

91. Arlet V, Marchesi D, Papin P, et al. Decompensation following scoliosis surgery: treatment by decreasing the correction of the main thoracic curve or "letting the spine go". Eur Spine J. 2000; 9(2):156-60.

92. Chang MS, Bridwell KH, Lenke LG, et al. Predicting the outcome of selective thoracic fusion in false double major lumbar "C" cases with five- to twenty-four-year follow-up. Spine. 2010; 35(24):2128-33.

93. Demura S, Murakami H, Kato S, et al. Spontaneous derotation of compensatory lumbar curve after thoracic fusion in adolescent idiopathic scoliosis. Spine Surg Relat Res. 2017;1(1):27-30.

94. Lee SM, Suk SI, Chung ER. Direct vertebral rotation: a new technique of three-dimensional deformity correction with seg- 
mental pedicle screw fixation in adolescent idiopathic scoliosis. Spine. 2004;29(3):343-9.

95. Behensky H, Cole AA, Freeman BJ, et al. Fixed lumbar apical vertebral rotation predicts spinal decompensation in Lenke type $3 \mathrm{C}$ adolescent idiopathic scoliosis after selective posterior thoracic correction and fusion. Eur Spine J. 2007;16(10):1570-8.

96. Singla A, Bennett JT, Sponseller PD, et al. Results of selective thoracic versus nonselective fusion in Lenke type 3 curves. Spine. 2014;39(24):2034-41.

97. Lonstein JE. Decompensation with Cotrel Dubousset instrumentation - a multicenter study. Orthop Trans. 1992;16:158.

98. Betz RR. Should all AIS $1 \mathrm{C}$ curves be fused selectively? Spine.
2016;41(7S):S16-7.

99. Studer D, Awais A, Williams N, et al. Selective fusion in adolescent idiopathic scoliosis: a radiographic evaluation of risk factors for imbalance. J Child Orthop. 2015;9(2):153-60.

100. Glassman SD, Bridwell K, Dimar JR, et al. The impact of positive sagittal balance in adult spinal deformity. Spine. 2005;30 (18):2024-9.

Spine Surgery and Related Research is an Open Access journal distributed under the Creative Commons Attribution-NonCommercial-NoDerivatives 4.0 International License. To view the details of this license, please visit (https://creativeco mmons.org/licenses/by-nc-nd/4.0/). 